\title{
Abelian monopole condensation in lattice gauge theories
}

\author{
P. Cea ${ }^{\mathrm{a}, \mathrm{b}}$, and L. Cosmai ${ }^{\mathrm{b}}$

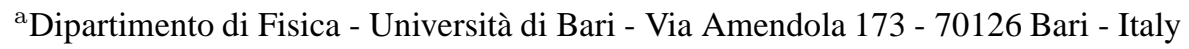 \\ ${ }^{\mathrm{b}}$ INFN - Sezione di Bari - Via Amendola 173 - 70126 Bari - Italy
}

We investigate the dynamics of lattice gauge theories in an Abelian monopole background field. By means of the gaugeinvariant lattice Schrodinger functional we study the Abelian monopole condensation in U(1) lattice gauge theory at zero temperature and in SU(3) lattice gauge theory at finite temperature.

\section{Introduction}

The dual superconductivity mechanism to explain color confinement has been suggested since the early day of QCD [1]. The first evidences for the dual superconductivity was obtained by studying the dual Meissner effect [2]. More recently an alternative method to detect the dual superconductivity has been proposed by the Pisa Group [3]: it consists in measuring a disorder parameter given in terms of an operator with non zero magnetic charge and nonvanishing v.e.v. in the confined phase. In the case of non Abelian Gauge theories they need to perform the Abelian projection. Indeed the Pisa Group found evidence of Abelian monopole condensation in several gauges: plaquette gauge, butterfly gauge and Polyakov gauge [4].

The aim of this work is to investigate the dynamics of lattice gauge theories in an Abelian monopole background field in a gauge-invariant way. We use the gauge-invariant effective action for external background field defined by means of the lattice Schrödinger functional [56]

$$
\mathcal{Z}\left[\vec{A}_{a}^{\mathrm{ext}}\right]=\int \mathcal{D} U \exp \left(-S_{W}\right)
$$

where $S_{\mathrm{W}}$ is the Wilson action and $\vec{A}^{\mathrm{ext}}(\vec{x})=$ $\vec{A}_{a}^{\text {ext }}(\vec{x}) \lambda_{a} / 2$ is the external field. The integration constraint over the lattice links is $\left.U_{\mu}(x)\right|_{x_{4}=0}=$ $U_{\mu}^{\text {ext }}(\vec{x}, 0)$, where $U_{\mu}^{\text {ext }}$ is the lattice version of the external field $A_{\mu}^{\text {ext }}$. The Schrödinger functional is invariant under arbitrary lattice gauge transformations of the boundary links. The lattice effective action for the background field $A_{\mu}^{\text {ext }}(\vec{x})$ is ( $L_{4}$ extension in Eu- clidean time):

$$
\Gamma\left[\vec{A}^{\mathrm{ext}}\right]=-\frac{1}{L_{4}} \ln \left\{\frac{\mathcal{Z}\left[U^{\mathrm{ext}}\right]}{\mathcal{Z}[0]}\right\}
$$

$\mathcal{Z}[0]$ is the lattice Schrödinger functional without external background field (i.e. $U_{\mu}^{\mathrm{ext}}=1$ ). Note that due to the manifest gauge invariance of the lattice background field effective action we do not need to fix the gauge.

\section{2. $\mathrm{U}(\mathbf{1})$}

We are interested in the effective action with a Dirac magnetic monopole background field. In the continuum the Dirac magnetic monopole field with the Dirac string in the direction $\vec{n}$ is:

$$
e \vec{b}(\vec{r})=\frac{n_{\mathrm{mon}}}{2} \frac{\vec{r} \times \vec{n}}{r(r-\vec{r} \cdot \vec{n})} .
$$

where, according to the Dirac quantization condition, $n_{\text {mon }}$ is an integer and $e$ is the electric charge (magnetic charge $=n_{\text {mon }} / 2 e$ ). We consider the gaugeinvariant background field action Eq. (2) where the external background field is given by the lattice version of the Dirac magnetic monopole field. In the numerical simulations we put the lattice Dirac monopole at the center of the time slice $x_{4}=0$. To avoid the singularity due to the Dirac string we locate the monopole between two neighbouring sites. We have checked that the results are not too sensitive to the precise position of the magnetic monopole. We introduce the disorder parameter for confinement:

$$
\mu=e^{-E_{\mathrm{mon}} L_{4}}=\frac{\mathcal{Z}[\mathrm{mon}]}{\mathcal{Z}[0]},
$$




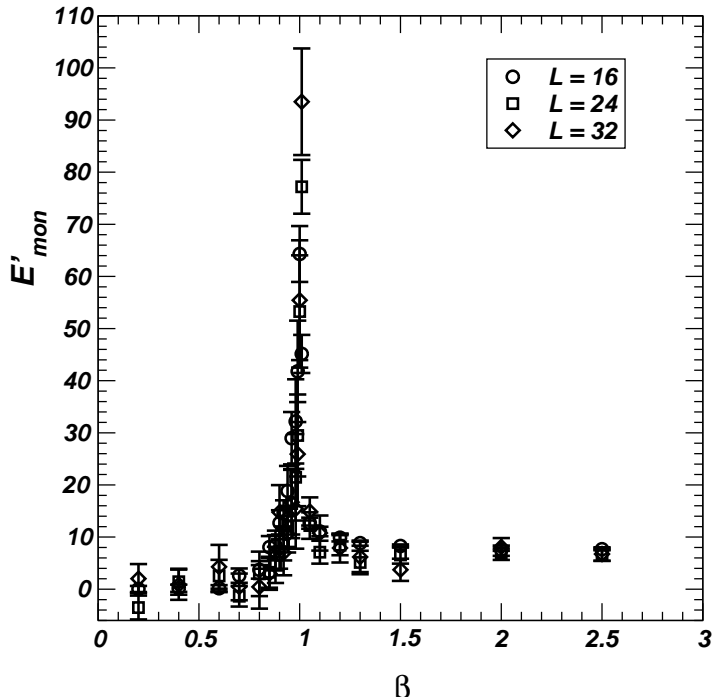

Figure 1. $E_{\text {mon }}^{\prime}$ for U(1) at three different lattice sizes.

where $\mathcal{Z}[0]$ is the Schrödinger functional with $n_{\text {mon }}=0$. According to the physical interpretation of the effective action, $E_{\text {mon }}$ is the energy to create a monopole. To avoid the problem of dealing with a partition function we consider $E_{\text {mon }}^{\prime}=\partial E_{\text {mon }} / \partial \beta$, that is analogous to the parameter $\rho$ introduced by the Pisa group [4]. Note that $E_{\text {mon }}^{\prime}$ is given by the difference between the average plaquette $P$ obtained from configurations without and with the monopole field.

We performed lattice simulations on $16^{4}, 24^{4}$ and $32^{4}$ lattices with periodic boundary conditions using the Quadrics/Q4 - QH1 in Bari. Note that the links belonging to the time slice $x_{4}=0$ and to the spatial boundary are constrained (no update). The constraint on the links starting from sites belonging to the spatial boundary corresponds in the continuum to the usual requirement that the fluctuations over the background field vanish at the spatial infinity. The contributions to $E_{\text {mon }}^{\prime}$ due to the constrained links must be subtracted, i.e.: only the dynamical links must be taken into account in evaluating $E_{\text {mon }}^{\prime}$. In the strong coupling region $\beta \lesssim 1$ the monopole energy is zero. This means that, according to Eq. (4), the disorder parameter $\mu \simeq 1$. Near the critical coupling $\beta_{c} \simeq 1, E_{\text {mon }}^{\prime}$ diplays a sharp peak which increases and shrinks by increasing the lattice volume. This means that the disorder parameter decreases towards zero in the thermodynamic limit when $\beta \rightarrow \beta_{c}$. In the weak coupling region $\left(\beta \gg \beta_{c}\right)$ the plateau in $E_{\text {mon }}^{\prime}$ indicates that the monopole energy tends to the classical monopole action which behaves linearly in $\beta$. In order to obtain $\mu$ we perform the numerical integration of $E_{\text {mon }}^{\prime}$ :

$$
E_{\mathrm{mon}}=\int_{0}^{\beta} E_{\mathrm{mon}}^{\prime} d \beta^{\prime}
$$

We found that the disorder parameter $\mu$ is different from zero in the confined phase (i.e. the monopoles condense in the vacuum). Moreover $\mu \rightarrow 0$ when $\beta \rightarrow \beta_{c}$ in the thermodynamic limit (the precise determination of $\beta_{c}$ require a F.S.S. analysis). Our result is gauge-invariant for the manifest gauge invariance of the Schrödinger functional.

\section{3. $\mathbf{S U}(3)$}

We have studied the Abelian monopole condensation in pure $\mathrm{SU}(2)$ lattice gauge theory at finite temperature. Here we restrict ourselves to the more interesting case of SU(3) gauge theory. In this case the maximal Abelian group is $\mathrm{U}(1) \times \mathrm{U}(1)$. Therefore we have two different types of Abelian monopole. Let us consider, firstly, the Abelian monopole field

$$
g \vec{b}^{a}(\vec{r})=\delta^{a, 3} \frac{n_{\mathrm{mon}}}{2} \frac{\vec{r} \times \vec{n}}{r(r-\vec{r} \cdot \vec{n})} .
$$

which we call the $T_{3}$-Abelian monopole. Now the functional integration constraint amounts on the lattice to fix the links belonging to the time slice $x_{4}=0$. In the present case the disorder parameter is defined as:

$$
\mu=e^{-F_{\mathrm{mon}} / T}=\frac{\mathcal{Z}[\mathrm{mon}]}{\mathcal{Z}[0]},
$$

where $T=1 / L_{t}$ is the temperature and $F_{\text {mon }}$ is the free energy per monopole. We measure $F_{\text {mon }}^{\prime}=$ $\partial F_{\text {mon }} / \partial \beta$. Again this corresponds to measuring the difference between the average plaquette without and with the monopole field.

From Fig. 2 we see that in the thermodynamic limit the disorder parameter $\mu \sim 1$ in the confined phase, moreover $\mu \rightarrow 0$ when $\beta \rightarrow \beta_{\mathrm{c}, \infty}=5.6925(2)$ [7] in the infinite volume limit.

The second type of Abelian monopole field is obtained from Eq. (6) replacing $\delta^{a, 3}$ with $\delta^{a, 8}$. A previous study [4] finds out that the disorder parameters for the two independent Abelian monopole defined by means of the Polyakov projection coincide 


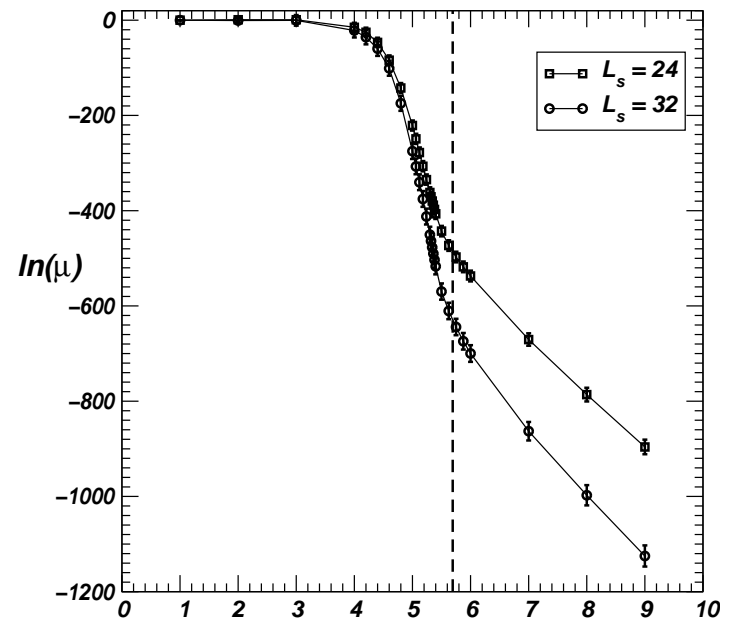

$\beta$

Figure 2. The logarithm of the disorder parameter $\mu$ for the SU(3) ( $T_{3}$ monopole) at two different spatial lattice sizes and $L_{t}=4$. Tha dashed line is the critical coupling.

within errors. On the contrary, our numerical results show a dramatic difference for $F_{\text {mon }}^{\prime}$. The peak of $F_{\text {mon }}^{\prime}$ in the case of the $T_{8}$-Abelian monopole is about an order of magnitude greater than in the $T_{3}$-Abelian monopole case (see Fig. 3). Consequently, in the former case, the disorder parameter $\mu$ tends to zero more sharply.

\section{Conclusions}

We have studied the Abelian monopole condensation both in the Abelian gauge theory U(1) and finite temperature non Abelian gauge theories SU(2) and SU(3). We introduce a disorder parameter which signals the Abelian monopole condensation in the confined phase. Our definition of the disorder parameter is by construction gauge invariant. Our numerical results suggest that the disorder parameter is different from zero in the confined phase and tends to zero when the gauge coupling $\beta \rightarrow \beta_{c}$ in the thermodynamic limit. Our estimate of the critical couplings are in fair agreement with the ones in the literarure. The precise determination of the critical couplings and the critical exponents in the infinite volume limit could be obtained by means of the finite size scaling analysis. In the case of the SU(3) gauge theory, there are two independent Abelian monopole fields

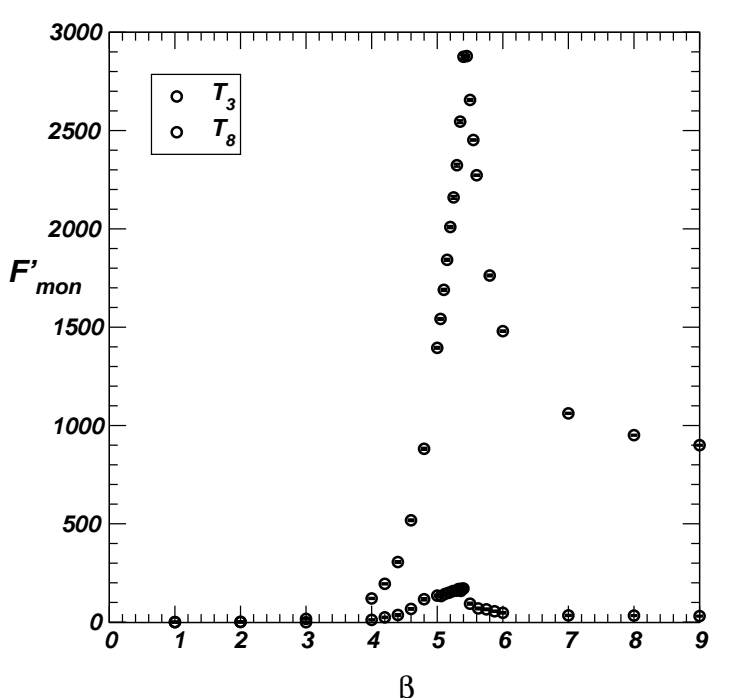

Figure 3. $F_{\text {mon }}^{\prime}$ for SU(3) for the $T_{3}$ and $T_{8}$ monopole on a $32^{3} \times 4$ lattice.

related to the two diagonal generators of the gauge group. Remarkably we find that the non perturbative vacuum reacts strongly in the case of $T_{8}$-Abelian monopole. This seems to suggest that the vacuum monopole condensate is predominantly formed by $T_{8}$ Abelian monopoles.

\section{REFERENCES}

1. G. 't Hooft, in "High Energy Physics", EPS Int. Conf., Palermo 1975, ed. A. Zichichi; S. Mandelstam, Phys. Rep. 23c, 245 (1976).

2. V. Singh, D.A. Browne and R.W. Haymaker, Nucl. Phys. Proc. Suppl. 30 (1993) 568; T. Suzuki, Nucl. Phys. Proc. Suppl. 30 (1993) 176; P. Cea and L. Cosmai, Nucl. Phys. Proc. Suppl. 30 (1993) 572; G.S. Bali, K. Schilling and C. Schlichter, Phys. Rev. D51 (1995) 5165.

3. L. Del Debbio, A. Di Giacomo and G. Paffuti, Phys. Lett. B349 (1995) 513.

4. A. Di Giacomo, B. Lucini, L. Montesi and G. Paffuti, hep-lat/9906024 hep-lat/9906025

5. G.C. Rossi and M. Testa, Nucl. Phys. B163 (1980) 109; Nucl. Phys. B176 (1980) 477.

6. M. Lüscher, R. Narayanan, P. Weisz, and U. Wolff, Nucl. Phys. B384, 168 (1992); M. Lüscher and P. Weisz, Nucl. Phys. B452, 213 (1995).

7. Y. Iwasaki et al., Phys. Rev. D46 (1992) 4657. 